\title{
EDITORIAL
}

\section{Consensus-based definition of unfitness to intensive and non- intensive chemotherapy in acute myeloid leukemia: a project of SIE, SIES and GITMO group on a new tool for therapy decision making}

Leukemia (2013) 27, 997-999; doi:10.1038/leu.2012.303

Acute myeloid leukemia (AML) is a disease of older adults, with a median age at diagnosis over 65 years. Studies from real-world practice $^{1}$ and experimental clinical trials ${ }^{2-4}$ have documented that age and frailty are prime predictors of outcome in AML. Accordingly, a proper assessment of patient's fitness represents a critical step in the therapeutic decision-making process of AML. ${ }^{5,6}$ Notwithstanding, physicians lack a common framework for establishing unfitness to different therapies in AML. A comprehensive evaluation of geriatric domains is time consuming and costly, and it measures characteristics that are not independently predictive of treatment toxicity, early mortality and overall survival in AML patients. More simple indexes, like the adapted Charlson comorbidity index ${ }^{7}$ and the hematopoietc cell transplantation comorbidity index, ${ }^{8}$ have an inherent risk of overemphasizing the measure of frailty of the patient with $A M L$ at diagnosis, being developed for patients in advanced stages of the disease.

In view of the clinical relevance of having a uniform and feasible definition of unfitness in $A M L$, we sought to provide such a definition by using the group and consensus methodologies under the auspices of the Italian Society of Hematology, Italian Society of Experimental Hematology (SIES) and Italian Group for Bone Marrow Transplantation (GITMO).

\section{THE CONSENSUS PROCESS}

We first recruited a panel of experts, defined as individuals with relevant knowledge, interest and skill in AML. We preferred a small group of dedicated experts than a larger one because of the narrow task, and the method used to reach the consensus.

Three orders of therapies were claimed to be worth to be distinguished in the treatment of patients with AML. Intensive chemotherapy was meant as any chemotherapeutic regimen aimed at achieving complete remission (CR), like combination of cytarabine and anthracyclines; non-intensive chemotherapy was as any therapy aimed at altering the natural course of the disease not necessarily achieving $C R$, such as low-dose cytarabine or hypomethylating agents; supportive therapy was meant as any therapy aimed at improving patient's quality of life, including cytoreductive therapies (hydroxyurea) and/or transfusion support.

Figure 1 shows the way the decision for selecting from the possible criteria could be framed as an analytic hierarchy process. ${ }^{9}$ It was agreed that four motivations should influence the decision of preferring one criterion to another for any definition of unfitness, namely: (a) to avoid therapies that could interfere with the age-dependent frailty of the patients; (b) to avoid therapies that could produce organ intolerance due to organ comorbidities; (c) to avoid therapies that the patient is unable to complain with, due to individual characteristics; and (d) to avoid risky therapies in patients whose life-expectancy is otherwise reduced by a nonAML disease.

We first aimed at selecting the criteria in their conceptual terms. To achieve this, a questionnaire was mailed to each member of the panel asking them to list candidate critical events they considered crucial for chemotherapy according to existing evidence and personal knowledge and experience. These criteria were further refined in a Delphi process. ${ }^{10}$ Candidate conceptual criteria were ranked according to their priority votes, with the criteria that ranked highest to be included in the list, forming the core set of conceptual criteria.

Using a bottom-up approach, the criteria were subjected to comparison according to their ability to fulfill one of the four motivations preliminarily selected for the decision according to the preferences of the members of the panel. This part of the process was exploited in a consensus meeting using the nominal group technique. ${ }^{11}$

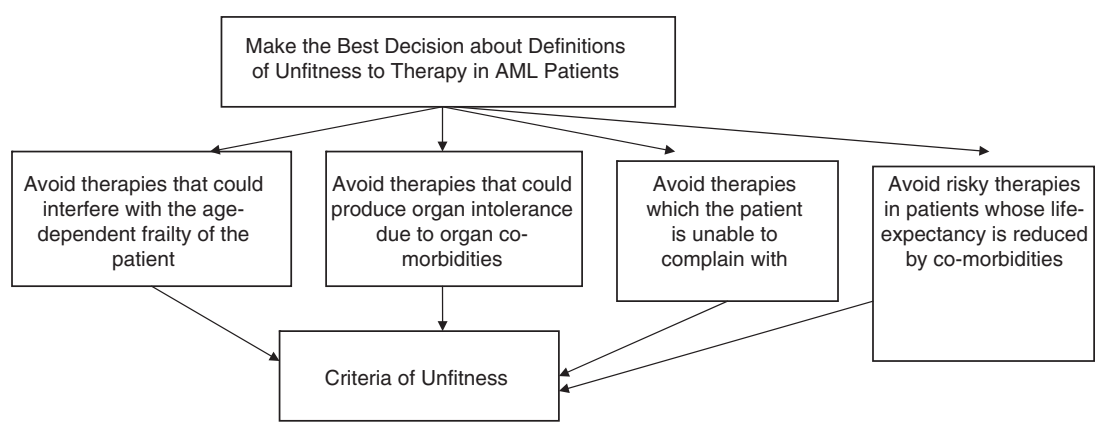

Figure 1. Analytic hierarchy process model regarding the selection of criteria for unfitness to therapy in patients with AML. At the top is the goal of the decision; at the bottom are the criteria to be decided; and in the middle are three motivations used for evaluating how well the options meet the goal. 
We then aimed at selecting the criteria in their operational terms populating them with quantitative or numerical attributes, or by categorizing the critical events that could exploit any conceptual criteria.

\section{THE DEFINITIONS OF UNFITNESS}

The panel listed 24 conceptual criteria to be included in the core set of candidate criteria for the definition of unfitness to intensive and non-intensive chemotherapy. The members of the panel

Table 1. Conceptual criteria to be used to define patients unfit to conventional intensive chemotherapy

1. $\quad$ Advanced age (over 75 years)

2. Severe cardiac comorbidity

3. Severe pulmonary comorbidity

4. Severe renal comorbidity

5. Severe hepatic comorbidity

6. Active infection resistant to anti-infective therapy

7. Cognitive impairment

8. Low performance status (ECOG functional scale)

9. Any other comorbidity that the physician judges to be incompatible with chemotherapy

Table 2. Conceptual criteria to be used to define patients unfit to non-intensive chemotherapy

\begin{tabular}{ll}
\hline 1. & Very severe cardiac comorbidity \\
2. & Severe pulmonary comorbidity \\
3. & Severe hepatic comorbidity \\
4. & Active infection resistant to anti-infective therapy \\
$5 .$. & Cognitive impairment \\
6. & Uncontrolled neoplasia
\end{tabular}

claimed that the cogent ailments were selected based on research of a number of authors, in which the criteria were selected based on their clinical implications for the newly diagnosed patients with AML. Using the pairwise comparisons, the members of the panel proposed that the definition of unfitness to intensive chemotherapy should require the fulfillment of at least one of nine criteria (Table 1) and that the definition of unfitness to non-intensive chemotherapy should require the fulfillment of at least one of six criteria (Table 2)

Finally, the panel listed the operational criteria to be included in the definitions of unfitness (Tables 3 and 4). The members of the panel claimed having largely derived operational criteria from the definitions of comorbidities from the literature with modifications.

\section{COMMENTS AND PERSPECTIVES}

To overcome the cognitive and practical issues inherent to the task of selecting criteria for unfitness to chemotherapy in AML, we used an approach based on analytic hierarchy process with multiple criteria process that reduces complex problems into small manageable parts. We provided definitions that consist in establishing the criteria widely used in different definitions of unfitness to therapies, but not in this precise combination and not as mutually exclusive categories.

An age older than 75 years was selected, reasoning that age is a strong determinant of host-specific clinical characteristics, such as cognitive, emotional and physical function, that predict vulnerability to toxicity.

Critical organs for comorbidity were selected among those scrutinized in the most used indices of comorbidities developed and evaluated in the effort to predict whether a patient is likely to do well after intensive chemotherapies.

Cognitive impairment may increase the risk of morbidity and mortality during therapy. ${ }^{12}$ In addition, it severely precludes patient/doctor interaction in the assessment of risk/benefit ratio

Table 3. Operation criteria to define unfitness to intensive chemotherapy in $\mathrm{AML}$

1. An age older than 75 years

2. Congestive heart failure or documented cardiomyopathy with an $\mathrm{EF} \leq 50 \%$

3. Documented pulmonary disease with DLCO $\leq 65 \%$ or FEV $1 \leq 65 \%$, or dyspnea at rest or requiring oxygen, or any pleural neoplasm or uncontrolled lung neoplasm

4. On dialysis and age older than 60 years or uncontrolled renal carcinoma

5. Liver cirrhosis Child B or C, or documented liver disease with marked elevation of transaminases ( $>3$ times normal values) and an age older than 60 years, or any biliary tree carcinoma or uncontrolled liver carcinoma or acute viral hepatitis

6. Active infection resistant to anti-infective therapy

7. Current mental illness requiring psychiatric hospitalization, institutionalization or intensive outpatient management, or current cognitive status that produces dependence (as confirmed by the specialist) not controlled by the caregiver

8. ECOG performance status $\geq 3$ not related to leukemia

9. Any other comorbidity that the physician judges to be incompatible with conventional intensive chemotherapy

Abbreviations: AML, acute myeloid leukemia; DLCO, diffusing capacity of the lungs for carbon monoxide; ECOG, Eastern Cooperative Oncology Group; $\mathrm{EF}$, ejection fraction; FEV1, forced expiratory volume in $1 \mathrm{~s}$.

Table 4. Operational criteria to define unfitness to non-intensive chemotherapy in AML

1. Refractory congestive heart failure

2. Documented pulmonary disease with DLCO $\leq 65 \%$ or FEV $1 \leq 65 \%$, or dyspnea at rest or requiring oxygen, or any pleural neoplasm or uncontrolled lung neoplasm

3. Liver cirrhosis Child $\mathrm{B}$ or $\mathrm{C}$ or acute viral hepatitis

4. Active infection resistant to anti-infective therapy

5. Current mental illness requiring psychiatric hospitalization, institutionalization or intensive outpatient management, or current cognitive status that produces dependence (as confirmed by the specialist) not controlled by the caregiver

6. Uncontrolled neoplasia

Abbreviations: AML, acute myeloid leukemia; DLCO, diffusing capacity of the lungs for carbon monoxide; ECOG, Eastern Cooperative Oncology Group; $\mathrm{EF}$, ejection fraction; FEV1, forced expiratory volume in $1 \mathrm{~s}$. 
and can motivate withholding of chemotherapy for the risk of treatment non-compliance.

Infections are an important cause of morbidity and mortality for patients with AML; therefore, the panel considered unresolved bacterial or fungal infections in the definition of unfitness in both intensive and, although at a lesser extent, non-intensive chemotherapy. $^{13}$

The performance status at AML presentation is associated with mortality within 30 days from induction. ${ }^{1,14}$ In the panelists' opinion, making a realistic judgment about patient's unfitness requires a careful performance status reassessment after adequate intensive supportive treatment. The threshold of performance status chosen for defining unfitness to intensive chemotherapy was a value $>2$ and the decision was in accordance with the results of the literature. ${ }^{1,15}$

Patient's financial and socioeconomic status, presence of a caregiver and distance from the caring center are further critical factors allowing care of AML to be delivered and follow-up to be performed. However, our panelists agreed that these dimensions should not entail a relevance, in the frame of the Italian perspective of social security and welfare, in that patients may trust in a number of facilities and supports (that is, home care, free transportation, social assistance).

In conclusion, we propose the use of novel definitions of unfitness to intensive and non-intensive chemotherapy in AML patients, which were developed using a analytic hierarchy process-based consensus process. We hope that adoption of these definitions will help the physician in the clinical practice of $A M L$, and will improve conduct and reporting of clinical trials. To enforce the purpose and aim of being a novel tool for improving the decision on chemotherapy in $A M L$, the results of this consensus work must be externally validated.

\section{CONFLICT OF INTEREST}

The authors declare no conflict of interest.

\section{ACKNOWLEDGEMENTS}

The funding of the project was provided by SIE, SIES and GITMO societies, and an arm's length contribution from Sanofi (Italy) was provided to SIE.

\footnotetext{
F Ferrara ${ }^{1}$, G Barosi ${ }^{2}$, A Venditti $^{3}$, E Angelucci ${ }^{4}$, M Gobbi $^{5}$, F Pane $^{6}$,

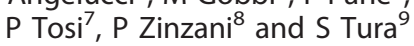

${ }^{1}$ Cardarelli Hospital, Division of Hematology and Stem Cell Transplantation Unit, Naples, Italy;

${ }^{2}$ Unit of Clinical Epidemiology and Center for the Study of Myelofibrosis, IRCCS Policlinico S, Matteo, Pavia, Italy;

${ }^{3}$ Department of Hematology, University Tor Vergata, Roma, Italy;

${ }^{4}$ Department of Medical Oncology, Ospedale Oncologico di Riferimento Regionale Armando Businco, Cagliari, Italy;

${ }^{5}$ Department of Hematology and Oncology, IRCCS Azienda Ospedaliera Universitaria S Martino-IST, Genova, Italy;
}

${ }^{6}$ CEINGE Biotecnologie Avanzate, Naples, Italy;

7Unit of Hematology, Ospedale Infermi, Rimini, Italy;

${ }^{8}$ Istituto di Ematologia ed Oncologia Medica 'Seragnoli', Università di Bologna, Bologna, Italy and ${ }^{9}$ University of Bologna, Bologna, Italy E-mail: felicettoferrara@katamail.com

\section{REFERENCES}

1 Juliusson G, Lazarevic V, Hörstedt AS, Hagberg O, Höglund M. Swedish Acute Leukemia Registry Group. Acute myeloid leukemia in the real world: why population-based registries are needed. Blood 2012; 119: 3890-3899.

2 Ferrara F, Palmieri S, Leoni F. Clinically useful prognostic factors in acute myeloid leukemia. Crit Rev Oncol Hematol 2008; 66: 181-193.

3 Malfuson JV, Etienne A, Turlure P, de Revel T, Thomas X, Contentin N et al. Acute Leukemia French Association (ALFA). Risk factors and decision criteria for intensive chemotherapy in older patients with acute myeloid leukemia. Haematologica 2008; 93: 1806-1813.

4 Djunic I, Virijevic M, Novkovic A, Novkovic A, Colovic N, Kraguljac-Kurtovic N et al. Comorbidity as a risk factor for overall survival and decision criteria for intensity of chemotherapy in elderly patients with acute myeloid leukemia. Med Oncol 2012; 29: 1077-1081.

5 Morra E, Barosi G, Bosi A, Ferrara F, Locatelli F, Marchetti M et al. Clinical management of primary non-acute promyelocytic leukemia acute myeloid leukemia: Practice guidelines by the Italian Society of Hematology, the Italian Society of Experimental Hematology, and the Italian Group for Bone Marrow Transplantation. Haematologica 2009; 94: 102-112.

6 Ferrrara F. Unanswered questions in acute myeloid leukemia. Lancet Oncol 2004; 5: 443-450.

7 Charlson ME, Pompei P, Ales KL, MacKenzie CR. A new method of classifying prognostic comorbidity in longitudinal studies: development and validation. J Chronic Dis 1987; 40: 373-383.

8 Sorror ML, Maris MB, Storb R, Baron F, Sandmaier BM, Maloney DG et al. Hematopoietic cell transplantation (HCT)-specific comorbidity index: a new tool for risk assessment before allogeneic HCT. Blood 2005; 106: 2912-2919.

9 Saaty TL, Vargas LG. Prediction, Projection And Forecasting: Applications of the Analytic Hierarchy Process in Economics, Finance, Politics, Games and Sports. Springer: New York, NY, 1990.

10 William PL, Webb C. The Delphi technique: a methodological discussion. J Adv Nurs 1994; 19: 180-186.

11 Delbecq AL, van de Ven AH, Gustafson DH. Group Techniques for Program Planning. A Guide to Nominal Group and Delphi Processes. Scott, Foresman and Co.: Glenview, IL, 1975.

12 Klepin HD, Balducci L. Acute myelogenous leukemia in older adults. Oncologist 2009; 14: 222-232.

13 Ostronoff $F$, Ostronoff $M$, Calixto R, Maior AP, Sucupira A, Florêncio $R$ et al. Successful nonmyeloablative allogeneic stem cell transplantation for therapyrelated acute myelogenous leukemia in a patient with preexisting visceral fungal infection. J Pediatr Hematol Oncol 2011; 33: 62-64.

14 Kantarjian H, O'brien S, Cortes J, Giles F, Faderl S, Jabbour E et al. Results of intensive chemotherapy in 998 patients age 65 years or older with acute myeloid leukemia or high-risk myelodysplastic syndrome: predictive prognostic models for outcome. Cancer 2006; 106: 1090-1098.

15 Colovic M, Colovic N, Radojkovic M, Stanisavljevic D, Kraguljac N, Jankovic G et al. Induction chemotherapy versus palliative treatment for acute myeloid leukemia in a consecutive cohort of elderly patients. Ann Hematol 2012; 91: 1363-1370. 